\title{
UNIT-REGULAR ORTHODOX SEMIGROUPS
}

\author{
by R. B. McFADDEN
}

(Received 5 May, 1983)

Introduction. Unit-regular rings were introduced by Ehrlich [4]. They arose in the search for conditions on a regular ring that are weaker than the ACC, DCC, or finite Goldie dimension, which with von Neumann regularity imply semisimplicity. An account of unit-regular rings, together with a good bibliography, is given by Goodearl [5].

The basic definition of unit-regularity is purely multiplicative; it is simply that for each element $x$ of a monoid $S$ (initially a ring $R$ with identity) there is a unit $u$ of $S$ for which $x=x u x$. The concept of a unit-regular semigroup is a natural one; for example, the full transformation semigroup on a finite set, and the semigroup of endomorphisms of a finite-dimensional vector space, are unit-regular semigroups [1]. Unit-regularity has been studied by Chen and Hsieh [2], by Tirasupa [9], and by McAlister [6]. The connection between unit-regularity and finiteness conditions has been considered by D'Alarcao [3].

The problem of describing the structure of an arbitrary unit-regular semigroup $S$ is difficult. It appears reasonable to attempt to provide such a description in terms of the group of units of $S$ and the set of idempotents of $S$, and in this direction Blyth and McFadden did determine the structure of a narrow class of unit-regular semigroups. Calling a semigroup $S$ uniquely unit orthodox if it is orthodox and, for each $x$ in $S$, there exists a unique unit $u$ of $S$ for which $x=x u x$, they proved that every such semigroup is a semidirect product of a group (the group of units of $S$ ) and a band (the band of idempotents of $S$ ).

In the present paper we shall show first that every unit-regular orthodox semigroup $S$ is an idempotent-separating homomorphic image of a uniquely unit orthodox semigroup (determined by the idempotents of $S$ and the units of $S$ ). The main result here is then the determination of all idempotent-separating congruences on uniquely unit orthodox semigroups.

1. Unit-regular semigroups. Much of what follows depends on the following elementary fact. If $S$ is a monoid and $g$ is a unit in $S$, then for each idempotent $e$ of $S$, $\mathrm{geg}^{-1}$ is an idempotent, and if $f$ in $S$ is also an idempotent then

$$
g(e f) g^{-1}=\left(\mathrm{geg}^{-1}\right)\left({\mathrm{g} f g^{-1}}^{-1}\right. \text {. }
$$

Definition 1.1. A monoid $S$ is said to be unit-regular if for each $x$ in $S$ there exists a unit $u$ of $S$ for which $x=x u x$.

For the rest of this paper we shall deal with unit-regular semigroups; each such is, of course, regular, though it is certainly not the case that $x=x u x$ implies that $u x u=u$. We shall denote by $E(=E(S))$ the set of idempotents of $S$ and by $G(=G(S))$ the group of units of $S$.

Clearly $x=x u x$ implies that $x u$ and $u x$ are idempotent, and each element of $S$ may

Glasgow Math. J. 25 (1984) 229-240. 
be written in the form $e g$ for $e$ in $E$ and $g$ in $G$. This factorization is not unique, though $e$ is unique for unit-regular inverse semigroups [2].

If $e g$ and $f h$, for $e, f$ in $E$ and $g, h$ in $G$, are elements of $S$ then

$$
e g f h=e\left(g f g^{-1}\right) g h=j k g h
$$

where $e, g f g^{-1}, j$ are idempotent and $k, g, h$ are units. Here

$$
j=j\left(e, g f g^{-1}\right) \in E \text { and } k=k\left(e, g f g^{-1}\right) \in G .
$$

Any useful description of the structure of $S$ in terms of $E$ and $G$ would need to provide more information about $j$ and $k$ than just the fact of their existence.

But there is one case in which $j$ and $k$ are obvious; if $S$ is orthodox ( $E$ is a subsemigroup of $S$ ) we can take $j=e\left(g f g^{-1}\right)$ and $k=1$, so that

$$
e g f h=e\left(g f g^{-1}\right) g h
$$

with $e\left(g f g^{-1}\right)$ in $E$ and $g h$ in $G$.

Definrmon 1.2. A monoid $S$ is said to be unit orthodox if $S$ is unit-regular and orthodox [1].

When $S$ is unit orthodox it is clear that $G$ acts automorphically (in the sense that there is a homomorphism from $G$ into the group of automorphisms of $E$ ) on the band $E$ of idempotents of $S$ under the action

$$
g . e=\operatorname{geg}^{-1} \text { for } g \text { in } G \text { and } e \text { in } E \text {. }
$$

As yet there is no method for describing the structure of a unit orthodox semigroup $S$ directly in terms of $E(S)$ and $G(S)$, but a method does exist for a sub-class of unit orthodox semigroups. Calling a monoid $S$ uniquely unit orthodox if for each $x$ in $S$ there is a unique unit $u$ in $S$ for which $x=x u x$, Blyth and McFadden proved the following theorem [1].

THEOREM 1.3. Let $E$ be a band with identity 1 and let $G$ be a group which acts automorphically on $E$. Denoting by $g$. $e$ the action of $g \in G$ on $e \in E$, define a product on $T=E \times G$ as follows:

$$
(a, g)(b, h)=(a(g . b), g h) .
$$

Then $T$ is a uniquely unit orthodox semigroup, which we shall denote by $E|\times| G$. Denoting also by 1 the identity of $G$,

$$
\begin{aligned}
& E(T)=\{(e, 1) \mid e \in E\} \sim E, \\
& G(T)=\{(1, g) \mid g \in G\} \sim G .
\end{aligned}
$$

If $x=(e, g)$ is an element of $T$ then the unique unit $u$ of $T$ for which $x=x u x$ is $u=\left(1, g^{-1}\right)$.

Conversely, every uniquely unit orthodox semigroup arises in this way.

It is worth noting, as pointed out in the proof of Theorem 1.3, that 1.e $e=e$ for each $e$ in $E$, and $g .1=1$ for each $g$ in G. 
While the class of uniquely unit orthodox semigroups is not a large one, it plays a decisive role in determining the structure of unit orthodox semigroups in general, as the following theorem shows.

THEOREM 1.4. Let $S$ be a unit orthodox semigroup with group of units $G$. For $e$ in $E=E(S)$ and $g$ in $G=G(S)$ define $g . e=g_{e} g^{-1}$. This action defines an automorphism of $E$, and the map which assigns this automorphism to $g$ is a homomorphism from $G$ into the group of automorphisms of $E$. If the uniquely unit orthodox semigroup $T=E|\times| G$ is defined as in Theorem 1.3, then the map $\theta: T \rightarrow S$ defined by $(e, g) \theta=e g$ is an idempotentseparating epimorphism.

Proof. As noted above, it is obvious that $G$ acts automorphically on $E$ under $g . e=g e g^{-1}$. For $a$ in $S$ choose a unit $g$ in $G$ such that $a=a g^{-1} a$, so that $e=a g^{-1} \in E$; then

$$
(e, g) \theta=e g=a g g^{-1}=a,
$$

so $\theta$ maps $T$ onto $S$.

If $(e, g)$ and $(f, h)$ are elements of $T$ then

$$
(e, g) \theta(f, h) \theta=e g f h=e\left(g f g^{-1}\right) g h=e(g . f) g h=((e, g)(f, h)) \theta,
$$

so $\theta$ is a homomorphism.

Finally, each idempotent of $T$ is of the form $(e, 1)$ for some $e$ in $E$, so if $(e, 1)$ and $(f, 1)$ are idempotents of $T$ satisfying $(e, 1) \theta=(f, 1) \theta$ then $e=e 1=f 1=f$; therefore $\theta$ is idempotent-separating, and the proof is complete.

Now that we know that every unit orthodox semigroup is an idempotent-separating homomorphic image of a uniquely unit orthodox semigroup, the question arises: What are the congruence relations on a uniquely unit orthodox semigroup which are contained in $\mathscr{H}$ ? We shall answer this question in Section 2.

2. The $\mathscr{D}$-class structure of $E|\times| G$. For any orthodox semigroup $S$ the finest inverse semigroup congruence $\mathcal{Y}$ on $S$ is given by $x$ Oy $w$ if and only if $V(x)=V(w)$ where $V(x)$ denotes the set of inverses of $x$. Further, on any band $E$ the $\mathscr{y}$-classes coincide with the $\mathscr{D}$-classes, and $E$ is a semilattice $Y$ of rectangular bands; in particular, if $x, y$, and $z$ are $\mathscr{D}$-equivalent elements of $E$ then $x y z=x z$. We shall write $D_{x}$ for the $\mathscr{D}=\mathscr{y}$-class of $x$ in $E$, and similarly for $\mathscr{R}$ and $\mathscr{L}$.

Now let $T$ be a uniquely unit orthodox semigroup. Since we need to know about congruence relations on $T$ contained in $\mathscr{H}$, we proceed to determine Green's relations on T. But while $\mathscr{H}$ determines the "local" properties it is $\mathscr{D}$ which determines the "global" properties of the congruences we are seeking.

By Theorem 1.3 we may assume that $T=E|\times| G$ under the operation

$$
(e, g)(f, h)=(e(g \cdot f), g h),
$$

where $E=E^{1}$ is a band with identity and $G$ is a group which acts automorphically on $E$. Recall that $1 . e=e$ and $g .1=1$ for each $e$ in $E$ and each $g$ in $G$. 
LEMmA 2.1. Let $(a, g)$ be an element of $T$. Then $(x, u)$ is an inverse of $(a, g)$ in $T$ if and only if $u=g^{-1}$ and $g . x \in D$ where $D$ is the $\mathscr{D}$-class of $a$ in $E$.

Proof. First,

$$
(a, g)(x, u)(a, g)=(a(g \cdot(x(u . a))), g u g)=(a, g)
$$

if and only if $u=g^{-1}$ and $a(g . x) a=a$, while

$$
\left(x, g^{-1}\right)(a, g)\left(x, g^{-1}\right)=\left(x\left(g^{-1} \cdot(a(g \cdot x))\right), g^{-1}\right)=\left(x, g^{-1}\right)
$$

if and only if $x\left(g^{-1} \cdot a\right) x=x$. Therefore $\left(x, g^{-1}\right)$ and $(a, g)$ are mutually inverse if and only if $a(g . x) a=a$ and $x\left(g^{-1} \cdot a\right) x=x$. Since $g$ acts as an automorphism, this is true if and only if $g . x$ is in $D$.

LEMMA 2.2. For each $e$ in $E$ and each $g$ in $G$,

$$
\text { g. } L_{e}=L_{\text {g.ee }}, \quad g \cdot R_{e}=R_{\mathrm{g} . e}, \quad g \cdot D_{e}=D_{\mathrm{g} . e} .
$$

Proof. This follows directly from the definition of Green's relations and the fact that $G$ acts automorphically.

Lemma 2.3. Let $(a, g),(b, h)$ be elements of $T$. Then

(i) $(a, g) \mathscr{R}(b, h)$ if and only if $a \mathscr{R} b$ in $E$.

(ii) $(a, g) \mathscr{L}(b, h)$ if and only if $\mathrm{g}^{-1} . a \mathscr{L} h^{-1} . b$ in $E$.

(iii) $(a, g) \mathscr{H}(b, h)$ if and only if $h g^{-1} . a \mathscr{D} a$ and $b=a\left(h g^{-1}, a\right)$.

Proof. (i) $(a, g) \mathscr{R}(b, h)$ if and only if there exist inverses $(a, g)^{\prime}$ of $(a, g)$ and $(b, h)^{\prime}$ of $(b, h)$ respectively for which $(a, g)(a, g)^{\prime}=(b, h)(b, h)^{\prime}$. By Lemma 2.1 this is true if and only if there exist $a^{\prime}$ in $V(a)$ and $b^{\prime}$ in $V(b)$ for which

$$
(a, g)\left(g^{-1} \cdot a^{\prime}, g^{-1}\right)=\left(a a^{\prime}, 1\right)=(b, h)\left(h^{-1} \cdot b^{\prime}, h^{-1}\right)=\left(b b^{\prime}, 1\right) ;
$$

in other words, if and only if $a \mathscr{R} b$ in $E$.

(ii) As in (i), $(a, g) \mathscr{L}(b, h)$ if and only if $g^{-1} \cdot a^{\prime} a=h^{-1} . b^{\prime} b$ for some $a^{\prime}$ in $V(a)$ and some $b^{\prime}$ in $V(b)$. But $a^{\prime} a \mathscr{L} a$ and $b^{\prime} b \mathscr{L} b$, while $g^{-1}$ and $h^{-1}$ preserve $\mathscr{L}$-classes by Lemma 2.2, and therefore $(a, g) \mathscr{L}(b, h)$ implies

$$
g^{-1} \cdot a \mathscr{L} g^{-1} \cdot a^{\prime} a=h^{-1} \cdot b^{\prime} b \mathscr{L} h^{-1} \cdot b .
$$

Conversely, $g^{-1} \cdot a \mathscr{L} h^{-1} . b$ implies

$$
g^{-1} \cdot\left(\left(g h^{-1} \cdot b\right) a\right)=\left(h^{-1} \cdot b\right)\left(g^{-1} \cdot a\right)=h^{-1} \cdot b,
$$

and certainly $h^{-1} \cdot b b=h^{-1} . b$, so with $a^{\prime}=g h^{-1} . b$ and $b^{\prime}=b$ we have $g^{-1} \cdot a^{\prime} a=$ $h^{-1} . b^{\prime} b$, so that $(a, g) \mathscr{L}(b, h)$.

(iii) By parts (i) and (ii) we have

$$
\begin{aligned}
(a, g) \mathscr{H}(b, h) & \text { if and only if } a \mathscr{R} b \text { and } g^{-1} \cdot a \mathscr{L} h^{-1} \cdot b \text { in } E \\
& \text { if and only if } a \mathscr{R} b \text { and } b \mathscr{L} h \mathrm{hg}^{-1} \cdot a \text { in } E \\
& \text { if and only if } \mathrm{hg}^{-1} . a \mathscr{D} a \text { and } b=a\left(h \mathrm{~g}^{-1} \cdot a\right),
\end{aligned}
$$


Corollary. Let $(a, 1)$ be an idempotent in $T$. Then $(b, h) \mathscr{H}(a, 1)$ if and only if $b=a(h . a)$ for $h . a \in D_{a}$.

The egg-box picture in $E$ when $(a, g) \mathscr{H}(b, h)$ in $T$ is as follows.

\begin{tabular}{l|c|c|c|c} 
& & & & \\
\hline & $a$ & & $b$ & \\
\hline & $g h^{-1} \cdot b$ & & & \\
\hline & & & $\mathrm{hg}^{-1} \cdot a$ & \\
\hline & & & &
\end{tabular}

Lemma 2.4. Let $D$ be a $\mathscr{D}$-class of $E$, let $a \in D$, and let $h \in G$. Then

$$
\text { h. } a \in D \text { if and only if } h . D=D \text {. }
$$

Proof. Suppose $h . a \mathscr{D} a$ and let $b \mathscr{D} a$. Then since $D$ is a rectangular band, $b=b a b$ and $a=a b a$. Applying $h$ to these equations yields $h . b \mathscr{D} h . a \mathscr{D} a$; that is, $h . D \subseteq D$.

Also, $a \mathscr{D} h . a$ implies that for some $c$ in $D$ we have $a \mathscr{R} c \mathscr{L} h . a$, so $h^{-1} \cdot a \mathscr{R} h^{-1} \cdot c \mathscr{L} a$; that is, $h^{-1} \cdot a \mathscr{D} a$. By the first part of the proof, $h^{-1} . D \subseteq D$ and therefore $D=1 . D \subseteq h . D$. Combining these inclusions yields the result.

Definition 2.5. For a $\mathscr{D}$-class $D$ of $E$ define

$$
S_{\mathrm{D}}=\{g \in G \mid \mathrm{g} \cdot \mathrm{D}=\mathrm{D}\} \text {. }
$$

Clearly $S$ is a subgroup of $G$, and by Lemma 2.4 ,

$$
g \in S_{D} \quad \text { if and only if } D \cap g . D \neq \varnothing .
$$

For each element $D$ of the semilattice of $\mathscr{D}$-classes of $E$ we now have a subgroup of $G$ which stabilizes $D$. These stabilizers are in fact intimately connected with the $\mathscr{H}$-classes, in particular with the maximal subgroups, of $T$.

Lemma 2.6. Let $(a, g)$ be an element of $T$ and let $D$ be the $\mathscr{D}$-class of $a$ in $E$. Then the $\mathscr{H}$-class of $(a, g)$ in $T$ is $\left\{(a(k . a), k g) \mid k \in S_{D}\right\}$.

Proof. By Lemma 2.3 we have

$$
\begin{aligned}
(a, g) \mathscr{H}(b, h) \quad & \text { if and only if } a \mathscr{R} b \text { and } \mathrm{g}^{-1} \cdot a \mathscr{L} h^{-1} \cdot b \\
& \text { if and only if } h g^{-1} \cdot a \mathscr{L} b \mathscr{R} a \text { and } h g^{-1}=k \in S_{\mathrm{D}} \\
& \text { if and only if } b=a(k \cdot a) \text { and } k \in S_{\mathrm{D}} .
\end{aligned}
$$

Therefore $(b, h)$ is in the $\mathscr{H}$-class of $(a, g)$ precisely when $(b, h)=(a(k . a), k g)$ for some $k$ in $S_{D}$.

LEMma 2.7. Let $(a, 1)$ be an idempotent in $T$. Then the maximal subgroup of $T$ containing $(a, 1)$ is isomorphic to $S_{D}$ where $D$ is the $\mathscr{D}$-class of a in $E$. 
Proof. Let $H$ denote the $\mathscr{H}$-class of $(a, 1)$. Define the mapping $\theta: H \rightarrow S$ by $(b, k) \theta=$ $k$, for $(b, k) \in H$. Clearly $\theta$ is injective; it is also surjective, because if $k \in S$ then $(a(k . a), k) \in H$ by Lemma 2.6. If $(b, k),(c, h) \in H$ then $b=a(k . a), c=a(h . a)$, again by Lemma 2.6, and so

$$
\begin{aligned}
(b, k)(c, h) & =(a(k . a), k)(a(h . a), h)=(a(k . a) k .(a(h . a)), k h) \\
& =(a(k . a)(k . a)(k h . a), k h)=(a(k h . a), k h),
\end{aligned}
$$

the last equality following from the fact that $D$ is a rectangular band of which $a, k . a$ and $k h . a$ are each elements. Therefore $\theta$ is a homomorphism, and the proof is complete.

3. Idempotent-separating congruences on $E|\times| G$. Consider an arbitrary orthodox semigroup $S$, and let $\rho$ be an idempotent-separating congruence on $S$. If $a$ and $b$ are elements of $S$ for which $a \rho b$ then for any inverse $a^{\prime}$ of $a$ there is an inverse $b^{\prime}$ of $b$ for which $a^{\prime} \rho b^{\prime}$. For certainly $a a^{\prime} \mathscr{R} b \mathscr{L} a^{\prime} a$, so there exists an inverse $b^{\prime}$ of $b$ in the $\mathscr{H}$-class of $a^{\prime}$ and since $a \rho b$ then $a a^{\prime}=b b^{\prime} \rho a b^{\prime}$, whence $a^{\prime} \rho a^{\prime} a b^{\prime}=b^{\prime} b b^{\prime}=b^{\prime}$. This is, of course, almost exactly the treatment provided by Meakin in [7], the only difference being that the choice of $a^{\prime}$ is at our disposal, a fact we shall use below to some advantage. Precisely as Meakin shows, the fact that $\rho$ is idempotent-separating implies that:

$$
\text { for each } x \text { in } E, \quad a^{\prime} x a=b^{\prime} x b \text { and } a x a^{\prime}=b x b^{\prime} \text {. }
$$

Let us apply this to the uniquely unit orthodox semigroup $T=E|\times| G$ defined as in Section 1 . First, it is easy to verify that $\left(g^{-1}, a, g^{-1}\right)$ is an inverse of the element $(a, g)$ of $T$. Therefore if $\rho$ is an idempotent-separating congruence on $T$ and $(a, g) \rho(b, h)$ then there exists an inverse $(b, h)^{\prime}$ of $(b, h)$ such that $(b, h)^{\prime} \rho\left(g^{-1} \cdot a, g^{-1}\right)$. By Lemma 2.1 we can take $(b, h)^{\prime}=\left(h^{-1} \cdot b^{\prime}, h^{-1}\right)$ for some $b^{\prime} \mathscr{D} b$. In fact, using Lemma 2.3(iii) and $\rho \subseteq H$ we have the $\mathscr{D}$-class picture

\begin{tabular}{l|c|c|c|c} 
& & & & \\
& $a$ & & $b$ & \\
\hline & $b^{\prime}$ & & $\mathrm{hg}^{-1} \cdot a$ & \\
\hline & & & &
\end{tabular}

and so $b^{\prime}=\left(h g^{-1} \cdot a\right) a$.

Applying to $T$ the property of $\rho$ noted above, it follows that for each $(x, 1)$ in $E(T)$, or equivalently, for each element $x$ of $E$,

$$
\begin{aligned}
& \left(g^{-1} \cdot a, g^{-1}\right)(x, 1)(a, g)=\left(h^{-1} \cdot b^{\prime}, h^{-1}\right)(x, 1)(b, h) \\
& (a, g)(x, 1)\left(g^{-1} \cdot a, g^{-1}\right)=(b, h)(x, 1)\left(h^{-1} \cdot b^{\prime}, h^{-1}\right)
\end{aligned}
$$

Evaluating these products and equating their first components yields:

If $(a, g) \rho(b, h)$ then for each $x$ in $E, a x a=g h^{-1} .\left(b^{\prime} x b\right)$ and $a(g \cdot x) a=b(h . x) b^{\prime}$ for some inverse $b^{\prime}$ of $b$. 
There is a dual condition based on the fact that $\left(h^{-1} \cdot b, h^{-1}\right)$ is an inverse of $(b, h)$; it is that:

If $(a, g) \rho(b, h)$ then for each $x$ in $E, b x b=h g^{-1} .\left(a^{\prime} x a\right)$ and $b(h . x) b=a(g . x) a^{\prime}$ for some inverse $a^{\prime}$ of $a$.

Any congruence relation $\rho$ on $T$, when restricted to a group $\mathscr{H}$-class of $T$, determines a normal subgroup of that maximal subgroup. Let $(a, 1) \in E(T)$ and denote by $D$ the $\mathscr{D}$-class of $E$ containing $a$. By Lemma 2.7 the $\mathscr{H}$-class of $(a, 1)$ is isomorphic to the subgroup $S_{\mathrm{D}}$ of $G$ which stabilizes $D$, and when $\rho$ is restricted to this maximal subgroup of $T$ it determines, by the second projection mapping, a normal subgroup $N_{a}$ of $S_{D}$, namely

$$
N_{a}=\left\{k \in S_{D} \mid(a(k . a), k) \rho(a, 1)\right\} .
$$

Given any two idempotents $(a, 1)$ and $(b, 1)$ of $T$, then since $\rho$ is a congruence and $(a, 1)(b, 1)=(a b, 1)$, it follows that

$$
N_{a} N_{b} \subseteq N_{a b}
$$

Since each $N_{x}$ is a subgroup of $G$ it is then the case that:

$$
\text { for each } a, x \text { in } E, \quad N_{a} \subseteq N_{a x} \text { and } \quad N_{a} \subseteq N_{x a} .
$$

In particular, when $a \mathscr{D} b$ in $E$, so that $a=a b a$ and $b=b a b$, we can conclude that $N_{a} \subseteq N_{a b} \subseteq N_{b a b}=N_{b}$, and similarly that $N_{b} \subseteq N_{a}$. Therefore $a \mathscr{D} b$ implies that $N_{a}=N_{b}=$ $N_{D}$, say, where $D$ is the $\mathscr{D}$-class of $a$ and $b$. We have therefore:

(1) for any two elements $D, D^{\prime}$ of the semilattice $\mathscr{Y}$ of $\mathscr{D}$-classes of $E$,

$$
N_{D} \subseteq N_{D^{\prime}} \quad\left(=N_{D^{\prime} D}\right) .
$$

Further, if $D$ is any $\mathscr{D}$-class of $E$, if $a \in D$, if $k$ is an arbitrary element of $G$, and $n \in N_{\mathrm{D}}$, then $(n(n, a), n) \rho(a, 1)$ implies

$$
(1, k)(n(n . a), n)\left(1, k^{-1}\right)=\left(\ldots, k n k^{-1}\right) \rho(1, k)(a, 1)\left(1, k^{-1}\right)=(k . a, 1) ;
$$

that is,

(2) for each $k$ in $G$ and for each $D$ in $\mathscr{Y}$,

$$
k N_{D} k^{-1} \subseteq N_{D^{\prime}} \quad \text { where } D^{\prime} \text { is the } \mathscr{D} \text {-class of } k . a \text {. }
$$

We are now in a position to state and prove the main result of the paper.

THEOREM 3.1. Let $T$ be a uniquely unit orthodox semigroup, say $T=E|\times| G$ as defined in Section 1. Suppose that for each element $D$ of the semilattice $\mathscr{Y}$ of $\mathscr{D}$-classes of $E$ we have a normal subgroup $N_{\mathrm{D}}$ of the stabilizer $S_{\mathrm{D}}$ of $D$, and that the collection of these normal subgroups satisfies (1) and (2) above. Define the relation $\sigma$ on $T$ by:

$$
(a, g) \sigma(b, h) \text { if and only if } h g^{-1} \in N_{D} \text {, }
$$

where $D$ is the $\mathscr{D}$-class of $a$ (and of $b$ ), and there exist $a^{\prime} \in V(a), b^{\prime} \in V(b)$ such that for 
each $x$ in $E$,

(3)

$$
\begin{array}{rlrl}
a x a & =g h^{-1} \cdot\left(b^{\prime} x b\right), & & a(g \cdot x) a=b(h \cdot x) b^{\prime}, \\
b x b & =h g^{-1} .\left(a^{\prime} x a\right), & b(h \cdot x) b=a(g \cdot x) a^{\prime} .
\end{array}
$$

Then $\sigma$ is an idempotent-separating congruence on $T$.

Conversely, every idempotent-separating congruence on a uniquely unit orthodox semigroup arises in this way.

Proof. We begin by showing that $\sigma \subseteq \mathscr{H}$. Suppose that $(a, g) \sigma(b, h)$ and that $a^{\prime} \in V(a)$ and $b^{\prime} \in V(b)$ satisfy (3). Setting $x=1$ in $a(g . x) a=b(h . x) b^{\prime}$ yields $a=b b^{\prime} \mathscr{R} b \in D$; setting $x=1$ in $b x b=h g^{-1} .\left(a^{\prime} x a\right)$ yields $b=h g^{-1} .\left(a^{\prime} a\right) \mathscr{L} h g^{-1} . a$, and so $h^{-1} . b \mathscr{L} \mathrm{g}^{-1}$.a. It follows from Lemma 2.3(iii) that $\sigma \subseteq H$.

It is obvious that $\sigma$ is reflexive and symmetric. For transitivity, suppose that $(a, g) \sigma(b, h)$ and $(b, h) \sigma(c, k)$. Then there exist $a^{\prime} \in V(a), b^{\prime}, b^{\prime \prime} \in V(b), c^{\prime} \in V(c)$ such that for each $x$ in $E$ we have (3) and

$$
\begin{array}{ll}
b x b=h k^{-1} .\left(c^{\prime} x c\right), & b(h . x) b=c(k \cdot x) c^{\prime}, \\
c x c=k h^{-1} .\left(b^{\prime \prime} x b\right), & c(k \cdot x) c=b(h . x) b^{\prime \prime} .
\end{array}
$$

First, $k^{-1} g=\left(k^{-1} h\right)\left(h^{-1} g\right) \in N_{D} N_{D}=N_{D}$. Next, we require the existence of $a^{\prime \prime}$ in $V(a), c^{\prime \prime}$ in $V(c)$ such that for each $x$ in $E$,

$$
\begin{aligned}
a x a & =g k^{-1} \cdot\left(c^{\prime \prime} x c\right), & a(g \cdot x) a=c(k \cdot x) c^{\prime \prime}, \\
c x c & =k g^{-1} \cdot\left(a^{\prime \prime} x a\right), & c(k \cdot x) c=a(g \cdot x) a^{\prime \prime} .
\end{aligned}
$$

The following configuration holds in $D$.

\begin{tabular}{l|c|c|c|c|c|c}
\hline & $a$ & & $b$ & & $c$ & \\
\hline & $g h^{-1} \cdot b$ & & $a^{\prime}$ & & & \\
\hline & $b^{\prime}$ & & $h g^{-1} \cdot a$ & & & \\
\hline & & & $c^{\prime}$ & & $k h^{-1} \cdot b$ & \\
\hline & $g k^{-1} \cdot c$ & & $h k^{-1} \cdot c$ & & $b^{\prime \prime}$ & \\
\hline & $c^{\prime \prime}$ & & & & $a^{\prime \prime}$ & \\
\hline & & & & & $k g^{-1} \cdot a$ & \\
\hline
\end{tabular}


Defining $a^{\prime \prime}$ and $c^{\prime \prime}$ by this configuration, for each $x$ in $E$,

$$
\begin{aligned}
c(k \cdot x) c^{\prime \prime} & =c(k \cdot x)\left(k^{-1} \cdot a\right) a \\
& =c\left(k \cdot\left(x\left(g^{-1} \cdot a\right)\right)\right) c a \text { since } a \mathscr{R} c \\
& =b\left(h \cdot\left(x\left(g^{-1} \cdot a\right)\right) b^{\prime \prime} a \text { using }(b, h) \sigma(c, k)\right. \\
& =b(h \cdot x)\left(h g^{-1} \cdot a\right) a \text { since } h g^{-1} \cdot a, b^{\prime \prime}, a \text { are all in } D \\
& =b(h \cdot x) b^{\prime}=a(g \cdot x) a \text { using }(a, g) \sigma(b, h) .
\end{aligned}
$$

Similarly, $a(g \cdot x) a^{\prime \prime}=c(k, x) c$. Also,

$$
\begin{aligned}
c^{\prime \prime} x c & =c^{\prime \prime} \text { axac } \quad \text { since } \quad c^{\prime \prime} \mathscr{L} a \mathscr{R} c \\
& =c^{\prime \prime}\left(a\left(g \cdot\left(\mathrm{g}^{-1} \cdot x\right)\right) a\right) c=c^{\prime \prime}\left(c\left(k \cdot\left(\mathrm{g}^{-1} \cdot x\right)\right) c^{\prime \prime}\right) c \\
& =c^{\prime \prime} c\left(\mathrm{~kg}^{-1} \cdot x\right) c^{\prime \prime} c=\left(\mathrm{kg}^{-1} \cdot a\right)\left(\mathrm{kg}^{-1} \cdot x\right)\left(\mathrm{kg}^{-1} \cdot a\right)=k \mathrm{~g}^{-1} \cdot(a x a),
\end{aligned}
$$

so $a x a=g k^{-1} \cdot\left(c^{\prime \prime} x c\right)$ and, similarly, $c x c=k g^{-1} \cdot\left(a^{\prime \prime} x a\right)$. Therefore $\sigma$ is an equivalence.

To show that $\sigma$ is compatible we show first that if $(a, g) \sigma(b, h)$ and $(c, k) \in T$ then $(c(k, a), k g) \mathscr{H}(c(k . b), k h)$, and dually for products on the right. Since $a \mathscr{R} b$ implies $k . a \mathscr{R} k . b$, and $\mathscr{R}$ is a left congruence, $c(k . a) \mathscr{R} c(k . b)$; by Lemma $2.3(\mathrm{i})$

$$
(c(k . a), k g) \mathscr{R}(c(k . b), k h) .
$$

For $\mathscr{L}$-equivalence, note that for each $x$ in $E$,

$$
\begin{aligned}
x a & =x a x a=x g h^{-1} \cdot\left(b^{\prime} x b\right) \text { using }(a, g) \sigma(b, h) \\
& =\left(x\left(g h^{-1} \cdot b^{\prime}\right)\right)\left(g h^{-1} \cdot x b\right) \mathscr{L} g h^{-1} . x b
\end{aligned}
$$

in $D_{b x}$, because $\mathscr{D}$ is a congruence and, using (1), $g h^{-1} \in N_{D} \subseteq N_{D^{\prime}} \subseteq S_{D^{\prime}}$ where $D^{\prime}$ is the $\mathscr{D}$-class of $x b$. Therefore $g^{-1} .(x a) \mathscr{L} h^{-1} .(x b)$, and in particular, setting $x=k^{-1} . c$, we have $g^{-1} \cdot\left(\left(k^{-1} \cdot c\right) a\right) \mathscr{L} h^{-1} \cdot\left(\left(k^{-1} \cdot c\right) b\right)$. By Lemma 2.3(iii) again, we obtain

$$
(c, k)(a, g) \mathscr{H}(c, k)(b, h) \text {. }
$$

For the products on the right by $(c, k)$,

$$
\begin{aligned}
(a(g . c))(b(h . c)) & =(a(g . c) a)(b(h . c)) \quad \text { since } a \mathscr{R} b \\
& =b(h . c) b^{\prime} b(h . c) \text { using }(a, g) \sigma(b, h) \\
& =(b(h . c))\left((h . c) b^{\prime}\right)(b(h . c)) \\
& =b(h . c),
\end{aligned}
$$

and similarly $b(h . c) a(g . c)=a(g . c)$, so $(a, g)(c, k) \mathscr{R}(b, h)(c, k)$. Finally,

$$
\begin{aligned}
g^{-1} \cdot a \mathscr{L} h^{-1} \cdot b & \Rightarrow a(g \cdot c) \mathscr{L}\left(g h^{-1} \cdot b\right)(g \cdot c)=g h^{-1} \cdot(b(h \cdot c)) \\
& \Rightarrow(g k)^{-1} \cdot(a(g \cdot c)) \mathscr{L}(h k)^{-1} \cdot(b(h \cdot c)),
\end{aligned}
$$

and so $(a, g)(c, k)$ and $(b, h)(c, k)$ are $\mathscr{L}$-equivalent, therefore $\mathscr{H}$-equivalent.

To prove that $\sigma$ is compatible with multiplication in $T$, let us continue to suppose that 
$(a, g) \sigma(b, h)$ and $(c, k) \in T$. We have the following $D$-class configurations.

\begin{tabular}{c|c|c|c|c}
\hline & $a$ & & $b$ & \\
\hline & $\mathrm{gh}^{-1} \cdot b$ & & $a^{\prime}$ & \\
\hline & $b^{\prime}$ & & $\mathrm{hg}^{-1} \cdot a$ & \\
\hline
\end{tabular}

\begin{tabular}{c|c|c|c|c} 
& & & \\
\hline & $a(g \cdot c)$ & & $b(h \cdot c)$ & \\
\hline & $g h^{-1} \cdot(b(h \cdot c))$ & & $(a(g \cdot c))^{\prime}$ & \\
\hline$(b(h \cdot c))^{\prime}$ & & $h \mathrm{~g}^{-1} \cdot(a(\mathrm{~g} \cdot c))$ & \\
\hline
\end{tabular}

Define $(a(g . c))^{\prime}$ and $(b(h . c))^{\prime}$ by the configuration on the right. Then

$$
\begin{aligned}
(a(g . c))^{\prime} & =\left(g h^{-1} \cdot(b(h . c))\right) b(h . c) \\
& =\left(g h^{-1} \cdot b\right) a(g . c) a b(h . c) \text { since } g h^{-1} \cdot b \mathscr{L} a \mathscr{R} b \\
& =\left(g h^{-1} \cdot b\right) b(h . c) b^{\prime} b(h \cdot c) \text { using }(a, g) \sigma(b, h) \\
& =\left(g h^{-1} \cdot b\right) b(h . c)(h \cdot c) b^{\prime} b(h . c) \\
& =\left(g h^{-1} \cdot b\right) b(h . c)=a^{\prime}(h . c)
\end{aligned}
$$

using $b(h . c) \mathscr{D}(h . c) b^{\prime}$. In the same way, $(b(h . c))^{\prime}=b^{\prime}(\mathrm{g} . c)$. Now for each $x$ in $E$,

$$
\begin{aligned}
(h k)(g k)^{-1} \cdot(a(g \cdot c))^{\prime} x a(g \cdot c) & =\left(h g^{-1} \cdot\left(a^{\prime}((h \cdot c) x) a\right)\right)(h \cdot c) \\
& =b(h \cdot c) x b b(h \cdot c),
\end{aligned}
$$

and similarly $(g k)(h k)^{-1} .\left((b(h . c))^{\prime} x b(h . c)\right)=a(g . c) x a(g . c)$. Further,

$$
\begin{array}{r}
a(g \cdot c)(g k \cdot x) a(g \cdot c)=a(g \cdot(c(k \cdot x))) a(g \cdot c)=b(h \cdot(c(k \cdot x))) b^{\prime}(g \cdot c) \\
=b(h \cdot c)(h k \cdot x) b(h \cdot c))^{\prime}
\end{array}
$$

and $b(h . c)(h k . x) b(h . c)=a(g . c)(g k . x)(a(g . c))^{\prime}$.

This completes the proof of compatibility on the right. For left compatibility, we note first that because $k N_{D} k^{-1} \subseteq N_{D^{\prime}}$ where $k . a \in D^{\prime}$, we can multiply on the left by the units $(1, k)$ or $\left(1, k^{-1}\right)$ respectively and observe that it is enough to prove

$$
\left.\left(\left(k^{-1} . c\right) a, g\right) \sigma\left(k^{-1} \cdot c\right) b, h\right) \text {. }
$$

Write $d=k^{-1} . c$ and note that $h \mathrm{~g}^{-1} \in N_{D_{d a}}$ because $N_{D} \subseteq N_{D_{d a}}$ by (1). Consider the configurations below.

\begin{tabular}{c|c|c|c|c} 
& & & \\
\hline & $a$ & & $b$ & \\
\hline & $g h^{-1} \cdot b$ & & $a^{\prime}$ & \\
\hline & $b^{\prime}$ & & $g h^{-1} \cdot a$ & \\
\hline
\end{tabular}

\begin{tabular}{c|c|c|c|c}
\hline & $d a$ & & $d b$ & \\
\hline & $g h^{-1} \cdot(d b)$ & & $(d a)^{\prime}$ & \\
\hline & $(d b)^{\prime}$ & & $\mathrm{hg}^{-1} \cdot(d a)$ & \\
\hline
\end{tabular}


Using these,

$$
\begin{aligned}
(d a)^{\prime} & =\left(g h^{-1} \cdot d\right)\left(g h^{-1} \cdot b\right)(a d a) b \text { since } d b=d a b, \quad a d a \mathscr{L} d a \mathscr{L}\left(g h^{-1} \cdot d\right)\left(g h^{-1} . b\right) \\
& =\left(g h^{-1} \cdot\left(d b b^{\prime} d d b\right)\right) b \text { using }(a, g) \sigma(b \cdot h) \\
& =\left(g h^{-1} \cdot(d b)\right) b \text { calculating in } D \text { and in } D_{d a} \\
& =\left(g h^{-1} \cdot d\right) a^{\prime} .
\end{aligned}
$$

Similarly $(d b)^{\prime}=\left(h g^{-1} . d\right) b^{\prime}$. It follows that for each $x$ in $E$,

$$
h g^{-1} .\left((d a)^{\prime} x d a\right)=h g^{-1} .\left(\left(g h^{-1} . d\right) a^{\prime} x d a\right)=d b x d b,
$$

and that $h g^{-1} .\left((d b)^{\prime} x d b\right)=d a x d a$. The other two equations in (3) of Theorem 3.1 also follow from $(d a)^{\prime}=\left(g h^{-1} . d\right) a^{\prime}$ and $(d b)^{\prime}=\left(h g^{-1} . d\right) b^{\prime}$. Therefore $\sigma$ is compatible, so is an idempotent-separating congruence on $T$.

Conversely, suppose that $\rho$ is an idempotent-separating congruence on $T$. We saw above that $\rho$ determines a collection of normal subgroups $N_{D}$, for $D$ in $\mathscr{Y}$, satisfying (1) and (2); let $\sigma$ denote the congruence determined by this collection as in the first part of the proof. If $(a, g) \sigma(b, h)$ then $h g^{-1} \in N_{D}$ implies that $\left(a\left(h g^{-1} . a\right), h g^{-1}\right) \rho(a, 1)$, and therefore $\left(b, h g^{-1}\right) \rho(a, 1)$ or, equivalently, $(b, h) \rho(a, g)$; that is $\sigma \subseteq \rho$. The reverse inclusion is obvious, so $\sigma=\rho$. This completes the proof of the theorem.

4. Examples. Theorem 1.3 enables us to construct all uniquely unit orthodox semigroups, Theorem 1.4 shows that every unit-regular orthodox semigroup is an idempotent-separating homomorphic image of one of the former, and Theorem 3.1 provides a description of the appropriate congruences. In practice, starting with a given group $G$ that acts automorphically on a band $E=E^{1}$, one would construct as above a unit-regular orthodox semigroup $T$ whose band is necessarily isomorphic to $E$, and usually one would like the group of units of $T$ to be isomorphic to $G$. To ensure isomorphism between the two groups one only has to take $N_{G}=\{1\}$ (denoting by $G$ the $\mathscr{D}$-class of 1 in $E$ ) in (1) and (2), and to notice for (1) that $G$ is the identity element of $\mathscr{Y}$, for (2) that $k .1=1$ for each $k$ in $G$.

A unit-regular orthodox semigroup $S$ constructed as in Section 3 by factoring via an idempotent-separating congruence on $T=E(S)|\times| G(S)$ will be an inverse semigroup precisely when $E=E(S)$ is a semilattice. In this case the inverse semigroup $T$ is the semi-direct product of a semilattice and a group. Every factorizable inverse semigroup [2] is of this sort [6], and may therefore be obtained by factoring $T$ by a congruence of the type described in Theorem 3.1. And when $E$ is a semilattice its $\mathscr{D}$-classes are singletons and the groups $S_{D}$ are the stabilizers of individual elements of $E$. The idempotentseparating congruences $\rho$ in this case may be defined much more simply than in Theorem 3.1 ; it is easy to see that:

$$
(a, g) \rho(b, h) \text { if and only if } a=b \text { and } h g^{-1} \in N_{b} \text {. }
$$

Chen and Hsieh [2] proved that each element of a factorizable inverse semigroup $S$ may be written in the form $x=e g$ for a unique $e$ in $E(S)$ and $g$ in $G$. This does not hold in general for unit-regular orthodox semigroups, as the example below shows. So while it is 
still true that each element is under a unit in the natural ordering, the uniqueness of $e$ in $x=e g$ has gone. Further, the natural ordering on a regular monoid $S$ is compatible with multiplication only if $S$ is an inverse semigroup [8].

EXAMPLE. Let $S$ be the semigroup of all $3 \times 3$ real matrices of the form

$$
\left[\begin{array}{lll}
1 & p & q \\
0 & 1 & r \\
0 & 0 & s
\end{array}\right]
$$

Then $S$ is a unit-regular orthodox semigroup with $E=E(S)$ consisting of the identity matrix and those matrices of $S$ for which $p=s=0$, and $G=G(S)$ those for which $s \neq 0$. The band of $S$ consists of two $\mathscr{D}$-classes, namely $\{1\}$ and one $\mathscr{R}$-class $D$, say, consisting of all the non-identity idempotents of $S$. By straightforward calculation, for each $e$ in $D$ the subgroup $N$ of units $g$ satisfying $e g e=e$ [1] consists of those units whose $(1,2)$-entry is zero, and $N$ is a normal subgroup of $G$. Each eSe except $S$ itself is isomorphic to the additive group of real numbers, and $S$ is the union of its group of units and its kernel, a single $R$-class consisting of the $e S e, e \in E-\{1\}$. There is no uniqueness of $e$ in $x=e g$ because $(E-\{1\}) N \subseteq E-\{1\}$.

By Theorem 1.4 the semigroup $S$ is the idempotent-separating homomorphic image of $T=E|\times| G$ under the mapping $(e, g) \mapsto e g$. Since the $\mathscr{D}$-class $D$ is a rectangular band $e T e=\{(a(g . a), \mathrm{g}) \mid \mathrm{g} \in G\} \cong G$ for each idempotent $e=(a, 1)$ with $a \in D$. An element $(a(g . a), g)$ maps to

$$
a(g \cdot a) g=a\left(\mathrm{gag}^{-1}\right) g=a g a .
$$

Therefore $(a(g . a), g)$ is congruent to $e$ if and only if the $(1,2)$-entry of $g$ is zero; that is, $N_{D}=N$. Therefore the congruence $\sigma$ defined in Theorem 3.1 is determined by just two normal subgroups of $G$, namely $N_{G}=\{1\}$ and $N_{D}=N$.

\section{REFERENCES}

1. T. S. Blyth and R. McFadden, Unit orthodox semigroups, Glasgow Math. J. 24 (1983), $39-42$.

2. S. Y. Chen and H. C. Hsieh, Factorizable inverse semigroups, Semigroup Forum 8 (1974), 283-297.

3. H. D'Alarcao, Factorizable as a finiteness condition, Semigroup Forum 20 (1980), 281-282.

4. G. Ehrlich, Unit-regular rings, Portugal. Math., 27 (1968), 209-212.

5. K. R. Goodearl, Von Neumann regular rings (Pitman, 1979).

6. D. B. McAlister, Some covering and embedding theorems for inverse semigroups, J. Austral. Math. Soc. Ser. A 22 (1976), 188-211.

7. J. C. Meakin, Congruences on orthodox semigroups, J. Austral. Math. Soc. 12 (1971), 323-341.

8. K. S. S. Nambooripad, Structure of regular semigroups. I, Mem. Amer. Math. Soc. 22 (1979), Number 224.

9. Y. Tirasupa, Factorizable transformation semigroups, Semigroup Forum 18 (1979), 15-20.

NORTHERN ILLINOIS UNIVERSITY

Dekalb, IL. 60115. 